\title{
On-the-fly Adiabatically Switched Semiclassical Initial Value Representation Molecular Dynamics for Vibrational Spectroscopy of Biomolecules
}

\author{
Giacomo Botti, Michele Ceotto, and Riccardo Conte* \\ Dipartimento di Chimica, Università degli Studi di Milano, via Golgi 19, 20133 Milano, Italy
}

Semiclassical (SC) vibrational spectroscopy is a technique capable of reproducing quantum effects (like zero-point energies, quantum resonances and anharmonic overtones) from classical dynamics runs even in the case of very large dimensional systems. In a previous work (R. Conte et al. J. Chem. Phys. 151, 214107 (2019)) a preliminary sampling based on adiabatic switching (AS) has been shown to be able to improve precision and accuracy of semiclassical results for challenging model potentials and small molecular systems. In this manuscript we investigate the possibility to extend the technique to larger (bio)molecular systems whose dynamics must be integrated by means of ab initio "on-the-fly" calculations. After some preliminary tests on small molecules, we obtain the vibrational frequencies of glycine improving on pre-existing SC calculations. Finally, the new approach is applied to 17-atom proline, an amino acid characterized by a strong intramolecular hydrogen bond.

\footnotetext{
* riccardo.conte1@unimi.it
} 


\section{INTRODUCTION}

A lot of attention in computational chemistry is with no surprise reserved to biological systems. These systems are challenging when it comes to describe their potential energy surface (PES) and simulate their molecular dynamics. As for the former aspect the basic and popular way to deal with it has been so far represented by force fields.[1-9] They have the advantage to make long-time simulations of large dimensional systems affordable. However, molecular mechanics force fields are based on a classical treatment of the electronic problem which leads to some poor accuracy in the description of important features, including hydrogen bonding. Advances in the field are currently being reported through the development of machine learning approaches and high-quality analytical PESs.[10-19] Similarly, progress on a quantum mechanical ground is much needed in dealing with the molecular dynamics and spectroscopy of biological systems. This is an emerging area of research, which is at the heart of the present manuscript and aims at advancing the current state-of-art limited to classical molecular dynamics for a better characterization of the vibrational features of biomolecules.

A practical way to add quantum effects to classical molecular dynamics simulations is represented by the Semiclassical initial value representation (SCIVR) molecular dynamics.[2029] The peculiar feature of SCIVR, which consists in approximating the quantum mechanical propagator starting from classical dynamical quantities collected along a trajectory, allows one to obtain a reliable spectroscopic description, including quantum effects, in principle for any kind of molecular system. Moving from the theoretical to the practical level one has to deal with a set of issues such as the necessity to slash computational times and the difficulty to get a sensible and reliable spectroscopic signal when studying large dimensional systems. Furthermore, it is necessary to keep the stability of the quantum (semiclassical) simulation in spite of the possible chaoticity of classical trajectories. We briefly note here that several semiclassical techniques have been developed over the years. They include: the multiple coherent semiclassical initial value representation (MC SCIVR),[30] which builds on previous research by Kaledin and Miller,[31, 32] and De Leon and Heller to obtain accurate spectroscopic results upon propagation of a single trajectory;[33] a divide-and-conquer semiclassical initial value representation (DC SCIVR) strategy to deal with the spectroscopy of high dimensional systems; [34-42] a combination of adiabatic switching[43-47] and SCIVR 
methods (AS SCIVR) to help overcome the issue of chaotic trajectories.[48]

Glycine, a building block of life and also the smallest among amino acids, is a relevant example of application of recent advances in both PES description and quantum (semiclassical) molecular dynamics for spectroscopic studies.[15, 49-51] A first semiclassical study of glycine by means of MC SCIVR was based on ab initio "on-the-fly" molecular dynamics.[52] It allowed two of us to investigate the very complex energy landscape of the four lowest-lying conformers of glycine dynamically, and corroborate previous findings of other static quantum approaches like vibrational self consistent field (VSCF) and second-order vibrational perturbation theory (VPT2). Very recently, a new technique for the creation of permutationally invariant PESs has permitted construction of a full-dimensional, very precise analytical surface for glycine on which eight conformers have been identified.[15] Both diffusion Monte Carlo (DMC) and AS-SCIVR calculations have been performed using this surface to better specify the link between the conformers through zero-point energy calculations. DMC and AS-SCIVR estimates were in excellent agreement (about 5 wavenumbers) and it was possible to conclude that the eight conformers can be grouped into four pairs of asymmetric double wells characterized by very low barriers between the two local minima.

Another amino acid of key interest is represented by proline. Proline is the only natural amino acid with a covalent bond between the side chain and the peptidic nitrogen. For this reason, it is properly classified as an imminoacid. Furthermore, the pyrrolidinic ring of proline introduces a rotational constraint on the $\mathrm{N}-\mathrm{C}^{\alpha}$ bond and reduces the conformational space of proline.[53,54] Structurally, proline contributes to the stabilization and folding of proteins. [54-58] The two low-energy proline conformers can be classified as cis and trans[59] based on the relative position of the $\mathrm{NH}$ bond and $\mathrm{COOH}$ group with respect to the ring plane. The relative stability of these two conformers has been subject of controversy, [59, 60] and a high-level vibrational spectroscopy study based on a quantum or semiclassical treatment of nuclei is missing.

Finally, we notice that SC spectroscopy has been so far employed also in other biological contexts, including protonated and neutral species, supramolecular systems, and nucleosides.[34, 49, 61-64]For this purpose the DC-SCIVR technique has been adopted with satisfactory and accurate results allowing for an easier assignment of spectral features. To this end, though, an improvement of spectral precision based on narrower signals is desirable, and application of AS SCIVR to very high dimensional systems is with no doubt a 
path worth being pursued. When performing semiclassical spectroscopy with multiple trajectories, AS strongly reduces the number of trajectories to be discarded, thus increasing the Monte Carlo integration efficiency. On the other hand, when running a single on-the-fly trajectory, the increased stability afforded by AS is expected to lead to the collection of more significant signal. In both cases, AS SCIVR is a useful tool to reduce the signal width and increase the spectrum accuracy.

The main goal of the manuscript is to investigate the possibility to interface AS SCIVR with ab initio "on-the-fly" molecular dynamics and check on the advantages that such an approach brings in when dealing with biomolecular systems. In doing this we also provide some preliminary evidence about the vibrational features of proline, which deserve a deeper elaboration in a future work. The manuscript is organized as follows: In Section II we briefly review the basics of SC molecular dynamics and the founding of the adiabatic switching technique. In Section III we focus on some preliminary tests on small molecules and then move to glycine and proline. Finally, some comments and future perspectives conclude the paper.

\section{METHODS}

\section{A. A brief review of the semiclassical approaches employed}

The hallmark of semiclassical molecular dynamics is represented by its capability of adding quantum features on top of affordable classical dynamics runs. The semiclassical vibrational power spectrum, $I(E)$, which provides an estimate of the quantum vibrational spectral density, can be obtained through Kaledin and Miller's time-averaged semiclassical initial value representation (TA SCIVR) formula:[31, 65-67]

$$
I(E)=\left(\frac{1}{2 \pi \hbar}\right)^{N_{v}} \iint d \mathbf{p}_{0} d \mathbf{q}_{0} \frac{1}{2 \pi \hbar T}\left|\int_{0}^{T} d t e^{\frac{i}{\hbar}\left[S_{t}\left(\mathbf{p}_{t}, \mathbf{q}_{t}\right)+E t+\phi_{t}\left(\mathbf{p}_{t}, \mathbf{q}_{t}\right)\right]}\left\langle\Psi \mid g_{t}\left(\mathbf{p}_{t}, \mathbf{q}_{t}\right)\right\rangle\right|^{2}
$$

where $E$ is the vibrational energy, $N_{v}$ is the number of vibrational degrees of freedom, $\left(\mathbf{p}_{0}, \mathbf{q}_{0}\right)$ are the starting conditions, $T$ is the total simulation time, $S_{t}\left(\mathbf{p}_{t}, \mathbf{q}_{t}\right)$ and $\phi_{t}\left(\mathbf{p}_{t}, \mathbf{q}_{t}\right)$ are the instantaneous classical action and prefactor phase respectively, and $\left\langle\Psi \mid g_{t}\left(\mathbf{p}_{t}, \mathbf{q}_{t}\right)\right\rangle$ is the quantum overlap between an arbitrary reference state $|\Psi\rangle$ and a coherent state evolved for a time $t\left(\left|g_{t}\right\rangle\right)$. Coherent states have the following Gaussian representation in the configuration 
space:[68-72]

$$
\left\langle\mathbf{q} \mid g_{t}\right\rangle=\left(\frac{\operatorname{det}(\Gamma)}{\pi^{N_{\nu}}}\right) \exp \left\{-\left(\mathbf{q}-\mathbf{q}_{t}\right)^{T} \frac{\Gamma}{2}\left(\mathbf{q}-\mathbf{q}_{t}\right)+\frac{i}{\hbar} \mathbf{p}_{t}^{T}\left(\mathbf{q}-\mathbf{q}_{t}\right)\right\},
$$

where $\Gamma$ is the coherent state width matrix, usually expressed as the diagonal matrix of the harmonic frequencies of vibration $\omega_{h o}$.

One computational bottleneck in evaluating Eq.(1) resides in the prefactor phase

$$
\phi_{t}=\text { phase }\left[\sqrt{\left|\frac{1}{2}\left(\frac{\partial \mathbf{q}_{t}}{\partial \mathbf{q}_{0}}+\Gamma^{-1} \frac{\partial \mathbf{p}_{t}}{\partial \mathbf{p}_{0}} \Gamma-i \hbar \frac{\partial \mathbf{q}_{t}}{\partial \mathbf{p}_{0}} \Gamma+\frac{i \Gamma^{-1}}{\hbar} \frac{\partial \mathbf{p}_{t}}{\partial \mathbf{q}_{0}}\right)\right|}\right]
$$

which requires evaluation of the monodromy matrix elements at each step along the dynamics. This matrix contains the phase space partial derivatives, it is also known as the stability matrix, and its time evolution demands for evaluation of the Hessian matrix $\mathbb{H}$ of the potential. To alleviate this major issue, the Hessian Database (HDB) method has been developed.[73] In this way the number of computed Hessians required for a satisfactory semiclassical spectrum can be largely reduced. In the on-the-fly implementation of HDB the trajectory file is scanned: when a geometry found along the trajectory is close to another one already present in the database (according to an arbitrary threshold parameter), then the Hessian corresponding to the latter is employed instead of evaluating a new one. In the case of a quasi-periodical trajectory, the number of required Hessian calculations can be reduced without any noticeable loss in the accuracy of results. [73]

Another major and expected computational bottleneck is represented by the large number of degrees of freedom one has to take into account when dealing with biomolecules. As a matter of fact, even the smallest amino acid, glycine, requires to treat 24 degrees of freedom. It is above any controversy that for spectroscopy applications it is necessary to be able to compute sensible spectroscopic signals and assign them to specific molecular vibrations even in the case of large molecules. SC molecular dynamics deals with all degrees of freedom on an equal ground, and in the applications here presented we were able to work in full dimensionality, including a 45-dimensional SC study of proline vibrational features.

However, as the system dimensionality increases, more and more refined techniques are necessary to keep the semiclassical calculation viable. Furthermore, analytical potential energy surfaces may be difficult to construct and are rarely available. In this case, the trajectory has to be run "on the fly" presumably employing density function theory (DFT) level of theory. This means that the single point energy is evaluated $a b$ initio for each 
step of the dynamics, further increasing the computational effort required. The multiple coherent semiclassical initial value representation (MC SCIVR) recipe is adopted to reduce the number of trajectories needed for a satisfactory semiclassical spectrum. [30, 52, 74, 75]

MC SCIVR is based on two pillars. First, trajectories are run at a tailored energy. This is usually given by a quantum harmonic estimate, which is easily available. De Leon and Heller demonstrated that if a single trajectory is run at the exact quantum energy of a state, then the SC formalism is able to return the exact solution.[33] Secondly, a tailored reference state is adopted with the effect of improving the signal-to-noise ratio of the vibrational mode under consideration. By means of MC SCIVR, one can obtain indeed reliable results by running just a single trajectory.[52] In the general case, this is represented by a trajectory with harmonic zero-point energy. If so, fundamental frequencies of vibration are found with very good accuracy. In the case in which a particular spectroscopic signal has to be refined, one is allowed to adopt the MC-SCIVR technique with a trajectory which has a harmonic quantum of excitation in the particular mode under refinement.

The on-the-fly adiabatic switching semiclassical initial value representation (otf-AS SCIVR) technique here proposed is specifically designed to improve upon the MC-SCIVR recipe. AS SCIVR has been recently developed and applied to small molecular systems for which a high-level analytical surface was available. [48] The method consists in switching the vibrational Hamiltonian of the system from the initial harmonic one $H_{\text {harm }}$ to the $a b$ initio anharmonic one $H_{\mathrm{anh}}$,

$$
H_{\mathrm{as}}=[1-\lambda(t)] H_{\mathrm{harm}}+\lambda(t) H_{\mathrm{anh}},
$$

where $\lambda$ is the following switching function chosen in agreement with the literature

$$
\lambda(t)=\frac{t}{T_{\mathrm{AS}}}-\frac{1}{2 \pi} \sin \left(\frac{2 \pi t}{T_{\mathrm{AS}}}\right),
$$

and $T_{\mathrm{AS}}$ is the total length of the adiabatic switching trajectory. If the switching is slow enough (ideally, infinitely slow), the classical adiabatic theorem guarantees that the action variables are conserved.[76] This means that the system can be prepared in any easily quantizable state (for instance an eigenstate of the harmonic Hamiltonian) and then adiabatically switched to arrive to an approximate quantization for the actual Hamiltonian, thus increasing the efficiency of initial condition sampling for the SC calculation. The adiabatic switching run is followed by the regular SC dynamics which is started from the final phase 
space coordinates obtained from the preliminary AS procedure $\left(\mathbf{p}_{a s}, \mathbf{q}_{a s}\right)$. By indicating the momenta and positions along this dynamics as

$$
\mathbf{p}_{t}^{\prime}\left(\mathbf{p}_{a s}, \mathbf{q}_{a s}\right), \mathbf{q}_{t}^{\prime}\left(\mathbf{p}_{a s}, \mathbf{q}_{a s}\right)
$$

we can write the AS-SCIVR working formula as

$$
I_{a s}\left(E ; \mathbf{p}_{a s}, \mathbf{q}_{a s}\right)=\left(\frac{1}{2 \pi \hbar}\right)^{N_{v}} \frac{1}{2 \pi \hbar T}\left|\int_{0}^{T} d t e^{\frac{i}{\hbar}\left[S_{t}\left(\mathbf{p}_{t}^{\prime}, \mathbf{q}_{t}^{\prime}\right)+E t+\phi_{t}\left(\mathbf{p}_{t}^{\prime}, \mathbf{q}_{t}^{\prime}\right)\right]}\left\langle\Psi\left(\mathbf{p}_{e q}, \mathbf{q}_{e q}\right) \mid g_{t}\left(\mathbf{p}_{t}^{\prime}, \mathbf{q}_{t}^{\prime}\right)\right\rangle\right|^{2},
$$

where the power spectrum now depends upon the AS conditions $\left(\mathbf{p}_{a s}, \mathbf{q}_{a s}\right)$, via the evolved positions and momenta $\left(\mathbf{p}_{t}^{\prime}, \mathbf{q}_{t}^{\prime}\right)$ in the instantaneous classical action, the phase prefactor and the coherent state $\left|g_{t}\right\rangle$. The reference state $|\Psi\rangle$ is built with equilibrium positions and momenta $\left(\mathbf{p}_{e q}, \mathbf{q}_{e q}\right)$, as in MC SCIVR:

$$
|\Psi\rangle=\prod_{j}^{N_{\mathrm{vib}}} \epsilon_{1, j}\left|\mathbf{p}_{e q, j}, \mathbf{q}_{e q, j}\right\rangle+\epsilon_{2, j}\left|-\mathbf{p}_{e q, j}, \mathbf{q}_{e q, j}\right\rangle
$$

where $N_{\mathrm{v}}$ is the number of vibrational degrees of freedom, $\left|\mathbf{p}_{e q, j}, \mathbf{q}_{e q, j}\right\rangle$ and $\left|-\mathbf{p}_{e q, j}, \mathbf{q}_{e q, j}\right\rangle$ are two coherent states, and $\epsilon_{1, j}$ and $\epsilon_{2, j}$ are the coefficients used to enforce parity. To obtain the complete vibrational spectrum, a single coherent state per degree of freedom must be employed. By using both $\epsilon_{1, j}$ and $\epsilon_{2, j}$ equal to $1 \forall j$, the zpe peak and even overtones are reproduced. The peak relative to the vibrational mode $j$ can be obtained by putting only $\epsilon_{2, j}=-1$. By managing the coherent states in the reference state, one is therefore capable to extract all vibrational features from a single trajectory.

\section{B. Computational Details}

A new ForTRAn90 code was written to include the adiabatic switching trajectory into the more general code previously developed in our group for application of "on-the-fly" SC molecular dynamics to spectroscopy. The new code interfaces with the open source NWCHEM electronic structure software to obtain ab initio energies and gradients. Initially,

a finite difference method was implemented, but it turned out to be too slow for practical use. Therefore, in the latest version of our code we required computation of the ab initio gradients directly to NwCHEM. This allows one to compute directly the analytical gradient, thus saving computational time. 
In SC molecular dynamics symplectic integrators are adopted to ensure high precision in the dynamics runs. One can commonly choose between two levels of complexity according to the usual trade-off between accuracy and computational costs. At a computationally cheaper level, the very popular velocity-Verlet algorithm is a second-order symplectic algorithm, while more refined 4-th order symplectic algorithms have also been developed. In the applications here presented, for the adiabatic switching dynamics we have employed the 4-th order symplectic algorithm for the smaller molecules, and the velocity-Verlet one for the larger systems. The SC dynamics, being performed on-the-fly with NWCHEM, is always based on the velocity-Verlet algorithm.

The initial conditions for the switching trajectory in mass-scaled phase space coordinates were randomly generated according to the following recipe based on action-angle coordinates

$$
\begin{cases}p_{0, i} & =-\sqrt{\left(2 n_{i}+1\right) \hbar \omega_{i}} \sin \left(2 \pi \zeta_{i}\right) \\ q_{0, i}-q_{e q, i} & =\sqrt{\frac{\left(2 n_{i}+1\right) \hbar}{\omega_{i}}} \cos \left(2 \pi \zeta_{i}\right),\end{cases}
$$

where $i$ indicates the generic $\mathrm{i}$-th vibrational degree of freedom, $n_{i}$ is the $i$-th vibrational quantum number, $\omega_{i}$ is the vibrational frequency of the $i$-th mode, $q_{e q, i}$ is the $i$-th massscaled equilibrium coordinate, and $\zeta_{i}$ is a variable which can be either randomly generated or set equal to $-1 / 4$ if one wants to start a single trajectory from the equilibrium geometry. In the case of a single trajectory, starting it from the equilibrium geometry (where normal modes are decoupled) it reduces mode coupling during the switching trajectory. Harmonic action variables are a straightfoward way to start the trajectory from harmonic quantization of energy. Furthermore, increasing the vibrational quantum number $n_{i}$ allows one to excite selectively the $i$-th normal mode, thus allowing for refinement of the associated semiclassical spectroscopic signal according to the MC-SCIVR approach.

At each step of the dynamics the system is evolved as follows:

1. The harmonic potential $V_{\text {harm }}$ and gradient $\mathbf{g}_{\text {harm }}$ are evaluated according to the initial phase-space coordinates;

2. Mass-scaled normal modes are mapped to Cartesian coordinates to write NWCHEM input;

3. The NWCHEM gradient task is launched; 
4. The ab initio energy and analytical gradient are read from the NWCHEM output file. The analytical gradient is transformed from Cartesian to mass-scaled normal modes;

5. The switching Hamiltonian $H_{\text {as }}$ and switching force $\dot{\mathbf{p}}$ are evaluated. The switching force is given by

$$
\dot{\mathbf{p}}=(\lambda-1) \mathbf{g}_{\mathrm{harm}}-\lambda \mathbb{C}^{T} \mathbf{g}_{a i}
$$

where $\mathbf{g}_{\text {harm }}$ is the analytical harmonic gradient, $\mathbb{C}^{T}$ is the transpose of the eigenvector matrix and $\mathbf{g}_{a i}$ is the mass-scaled ab initio Cartesian gradient;

6. The system is evolved with the chosen integration algorithm.

This iterative procedure is represented in Figure 1. Due to the constant back and forth from normal modes to Cartesian coordinates, the switching trajectory takes a little more time than an equal-length standard trajectory performed with NWCHEM.

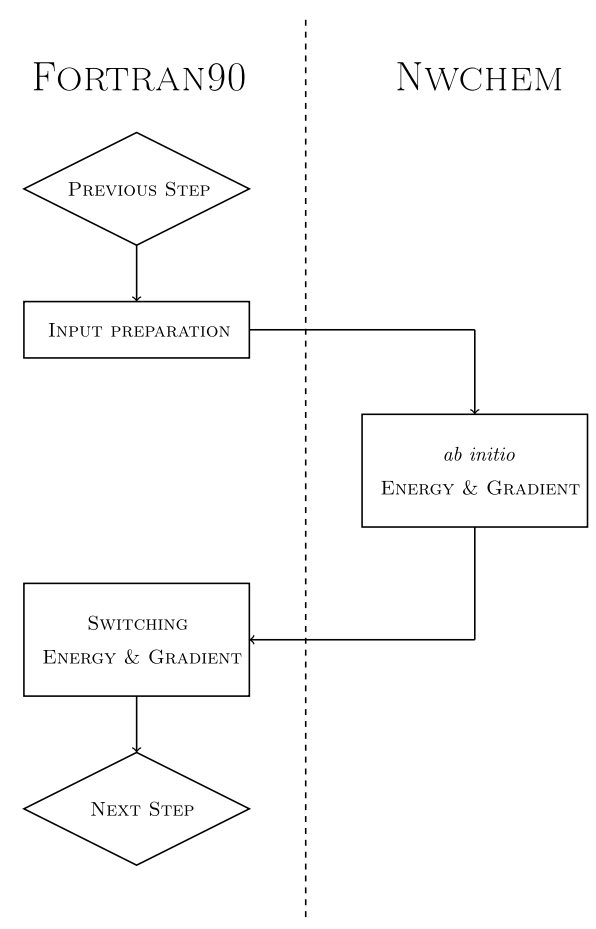

FIG. 1. Flux diagram of a single step of the Adiabatic Switching method

The code allows one to perform the on-the-fly Adiabatic Switching method. In this way, the usual semiclassical approach can be initiated from anharmonic conditions. Thus, the whole semiclassical procedure adopted in this paper (including adiabatic switching) is as follows: 
1. Preparation: the system is prepared in harmonic state $\left(\mathbf{p}_{\text {harm }} ; \mathbf{q}_{\text {harm }}\right)$;

2. Adiabatic Switching: the harmonic state is evolved under the switching hamiltonian Eq.(4), reaching the anharmonic state $\left(\mathbf{p}_{\mathrm{as}} ; \mathbf{q}_{\mathrm{as}}\right)$;

3. Classical Dynamics: the anharmonic state $\left(\mathbf{p}_{\mathrm{as}} ; \mathbf{q}_{\mathrm{as}}\right)$ is evolved with classical molecular dynamics, with an electronic structure software (NWCHEM);

4. TA-SCIVR: the classical action and Hessian matrices are computed from the classical trajectory, and the TA-SCIVR power spectrum, now more properly named AS-TA SCIVR, is obtained via Eq.(7). Apart from the working formula, this step has not changed from the previous semiclassical applications.

\section{RESULTS}

\section{A. Preliminary tests}

Some preliminary tests were performed on several molecules to study the efficiency and accuracy of different trajectory integration methods. The efficiency has been evaluated by estimating, for both the symplectic second (velocity Verlet) and fourth order integrators, the ratio of cpu times between a 2500-step AS trajectory and an equal length, velocityVerlet-integrated classical dynamics trajectory. Trajectories were evolved "on-the-fly" with NWCHEM. Results are reported in Table I, from which it is clear that, not surprisingly, the fourth order symplectic algorithm is substantially slower than the second order one. Very promisingly for the application of AS SCIVR to large molecules, if the same velocity Verlet integrator is used, then the AS procedure requires just a fraction of additional computational effort.

The accuracy of the integrator has been evaluated in terms of conservation of energy in an "on-the-fly" classical dynamics run at $H_{\text {harm }}$ energy, that is by using Eq.4 with $\lambda=0$. The relevant results are reported in Table II. These include the total energy of the test trajectory, chosen to be equal to the harmonic zero-point energy, and the standard deviation of the total energy calculated from the energy data of each step along the test trajectory. From the reported data it is evident that the fourth order is much more accurate than 
TABLE I. Ratio between the cpu time $t$ for an AS trajectory with second (superscript (2)) and fourth order (superscript (4)) simplectic algorithm, and an equal length NWCHEM molecular dynamics (MD) trajectory. In the last column, the ratio of AS cpu times is shown.

\begin{tabular}{c|ccc}
\hline Molecule & $t_{A S}^{(2)} / t_{M D}$ & $t_{A S}^{(4)} / t_{M D}$ & $t_{A S}^{(4)} / t_{A S}^{(2)}$ \\
\hline \hline $\mathrm{H}_{2}$ & 1.20 & 5.41 & 4.51 \\
$\mathrm{H}_{2} \mathrm{CO}$ & 1.12 & 5.90 & 5.00 \\
Gly & 1.41 & 6.26 & 4.44 \\
\hline
\end{tabular}

velocity Verlet whose accuracy is comparable only if a time step reduced by one order of magnitude is adopted.

TABLE II. Standard deviation $\sigma$ of the trajectory total energy. The subscript stands for the time step length in atomic units, while the superscript indicates the order of the symplectic integrator employed - (2) for second order, (4) for fourth order.

\begin{tabular}{c|c|cc|l}
\hline Molecule & $E_{\mathrm{zpe}}^{\mathrm{harm}} / \mathrm{cm}^{-1}$ & $\sigma_{1}^{(2)} / \mathrm{cm}^{-1} \sigma_{10}^{(2)} / \mathrm{cm}^{-1}$ & $\sigma_{1}^{(4)} / \mathrm{cm}^{-1} \sigma_{10}^{(4)} / \mathrm{cm}^{-1}$ \\
\hline \hline $\mathrm{H}_{2}$ & 2178 & 0.075 & 7.649 & $1.109 \times 10^{-7} 1.125 \times 10^{-3}$ \\
$\mathrm{H}_{2} \mathrm{CO}$ & 5780 & 0.011 & 3.180 & $6.915 \times 10^{-9} 2.070 \times 10^{-4}$ \\
$\mathrm{Gly}$ & 17336 & 0.078 & 8.650 & $7.109 \times 10^{-8} 8.260 \times 10^{-4}$ \\
\hline
\end{tabular}

The difference in the energy conservation properties of the two integrators is related to the different magnitudes of the typical oscillations in total energy of an adiabatic switching procedure. This is evident from the energy profile for the "on-the-fly" adiabatic switching dynamics performed for $\mathrm{H}_{2}$ and reported in Figure 2. The fourth order integrator is clearly more accurate and for this reason we employed it in our "on-the-fly" AS-SCIVR tests on small molecules described further below.

We conclude this Section by remarking that, with no surprise, the most efficient way to obtain the $a b$ initio gradient is by means of the NWCHEM[77] analytical gradient. This is doable for some levels of electronic theory including DFT. In this way, there is no need to employ a finite difference algorithm, which would require a number of single point energy calculations proportional to the number of vibrational normal modes. If this latter strategy had to be employed, then the cpu time for an "on-the-fly" AS trajectory would skyrocket even for small systems. By using the analytical gradient instead, the ratio between the AS 


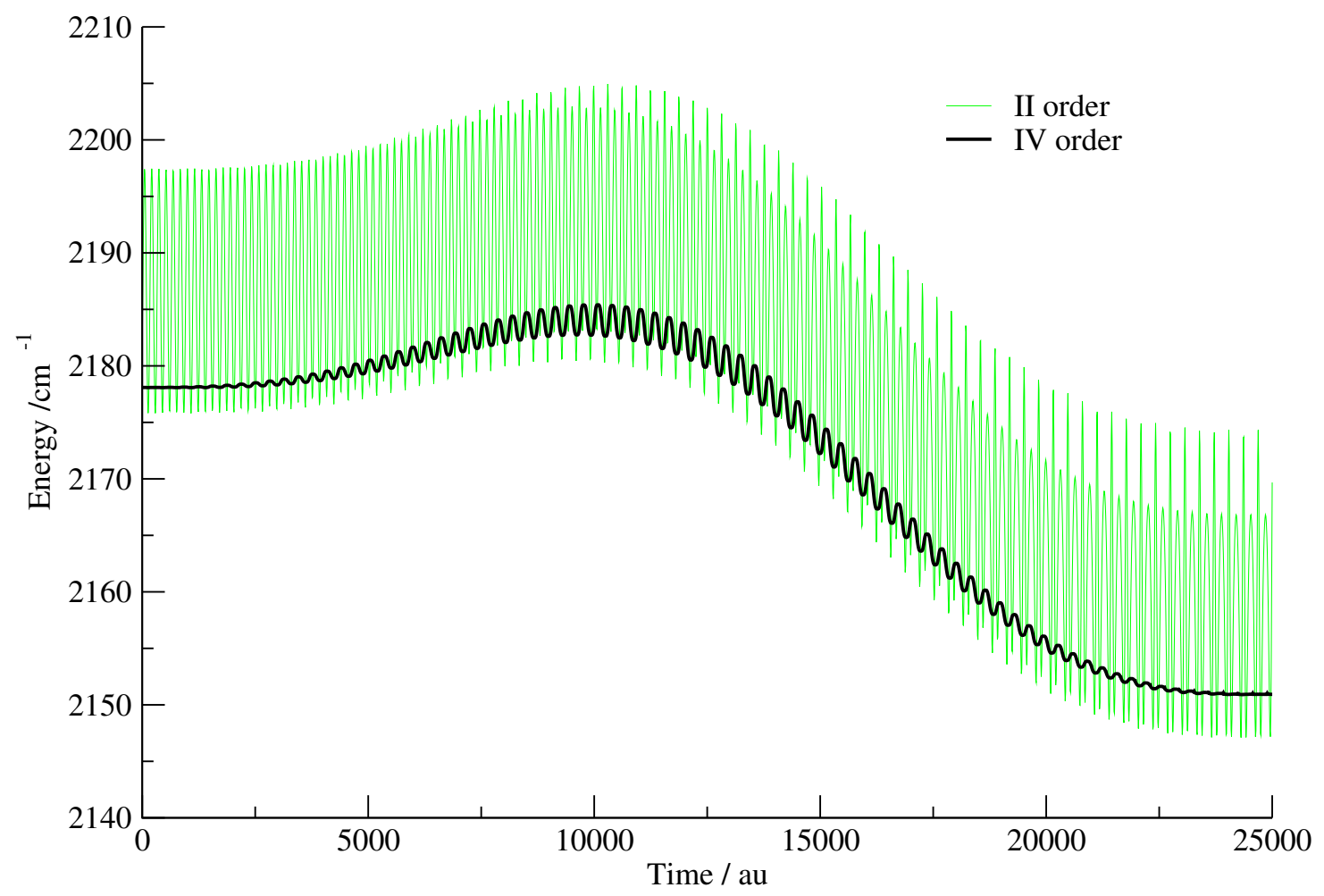

FIG. 2. Energy profile for the adiabatic switching trajectory of $\mathrm{H}_{2}$. The trajectories were integrated for a total of 2500 steps of 10 au each at DFT/B3LYP aug-cc-pVDZ level of theory

and standard Hamiltonian dynamics cpu time is basically constant and barely depends on the number of vibrational normal modes. See the Supplemental Material for relevant data on this aspect.

\section{B. Small Molecules}

The accuracy of the otf-AS-SCIVR method was first tested on small molecules, i.e. $\mathrm{H}_{2}$ and $\mathrm{H}_{2} \mathrm{CO}$, for which MC SCIVR is already known to provide satisfactory results. To this goal, as anticipated, we ran a preliminary 25000 au-long AS trajectory initiated from harmonic ZPE conditions. This was followed by a 25000 au-long classical trajectory for the SCIVR part of the simulation started from the final phase space coordinates of the AS trajectory. Both simulations were carried out at DFT/B3LYP level of theory with aug-cc-pVDZ basis set. The resulting AS-SCIVR spectra can be compared with MC-SCIVR ones, obtained with an equal length trajectory at same level of electronic theory but started from harmonic conditions. Figure 3 shows the comparison. The two spectra are pretty similar, as confirmed 
by the calculated frequency values. We looked at zero point energies, as well as fundamental and overtone frequencies. The zpe values are 2136 and $2127 \mathrm{~cm}^{-1}$ (corresponding to the two most intense signals in the two power spectra), the fundamental transition frequencies are 4158 and $4143 \mathrm{~cm}^{-1}$, and the overtone frequencies are 8429 and $8395 \mathrm{~cm}^{-1}$ for AS SCIVR and MC SCIVR, respectively. Detailed numerical values can be found in Table III.

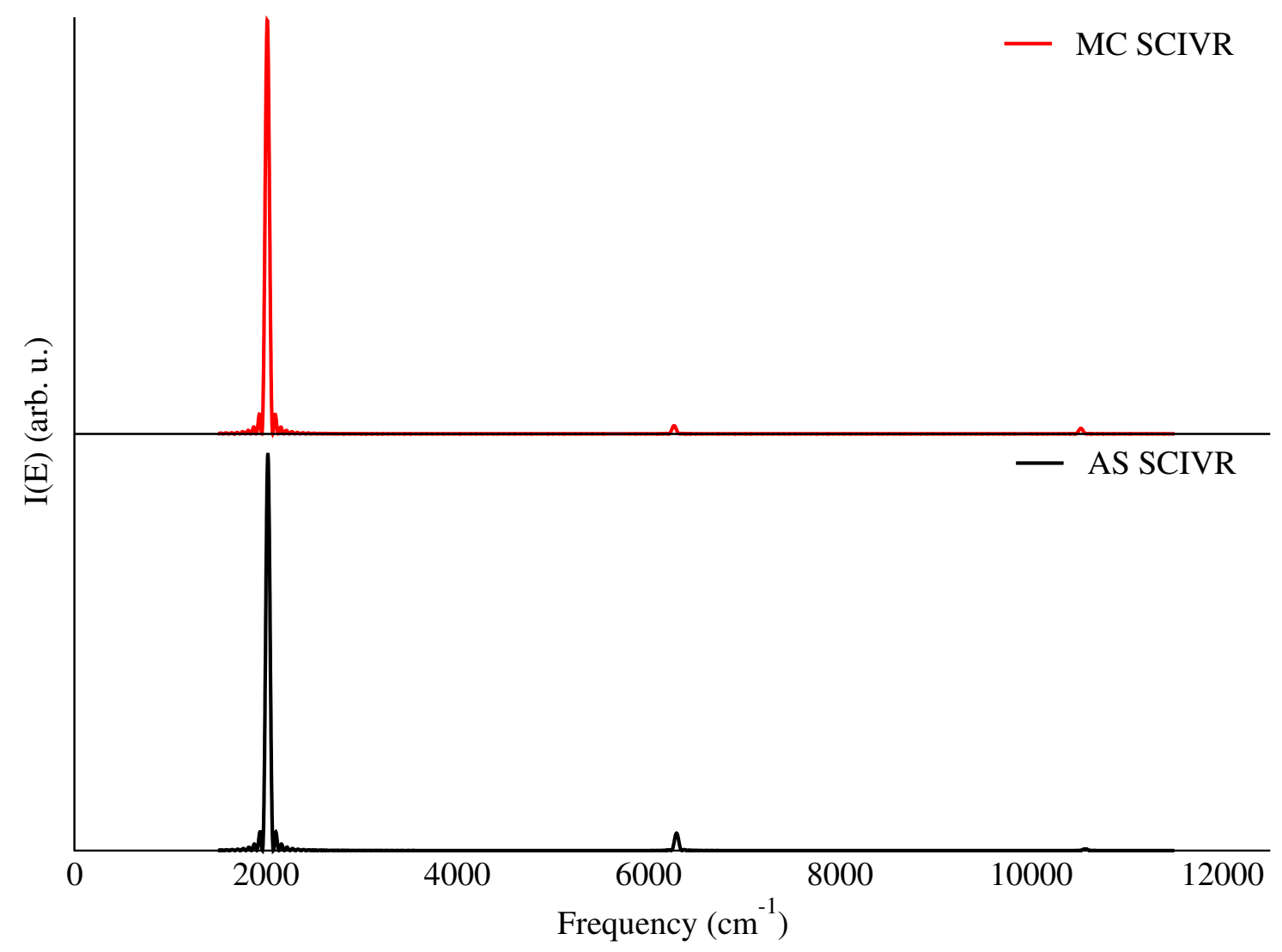

FIG. 3. MC-SCIVR (black, top) and AS-SCIVR (blue, bottom) power spectra for $\mathrm{H}_{2}$ based on single trajectories at DFT/B3LYP level of theory.

Afterwards, the vibrational power spectra of $\mathrm{H}_{2} \mathrm{CO}$ were obtained with AS SCIVR and MC SCIVR, following the same procedure as for $\mathrm{H}_{2}$ and employing the same trajectory length and level of electronic structure theory. The spectra of the different modes of $\mathrm{H}_{2} \mathrm{CO}$ were compared between the two methods and the available experiments. Results are given in Figure 4.

The theoretical results are in excellent agreement between each other and the mean absolute error (MAE) with respect to the experiment is below $20 \mathrm{~cm}^{-1}$ in spite of the DFT level of theory employed. The spectroscopic signals for this small molecule have comparable 
widths, with MC-SCIVR peaks slightly narrower. This is unexpected because AS SCIVR has been demonstrated to provide narrower peaks, a feature that will be clearly pointed out moving to larger molecules. However, this turns out to be a fictitious feature. In fact,

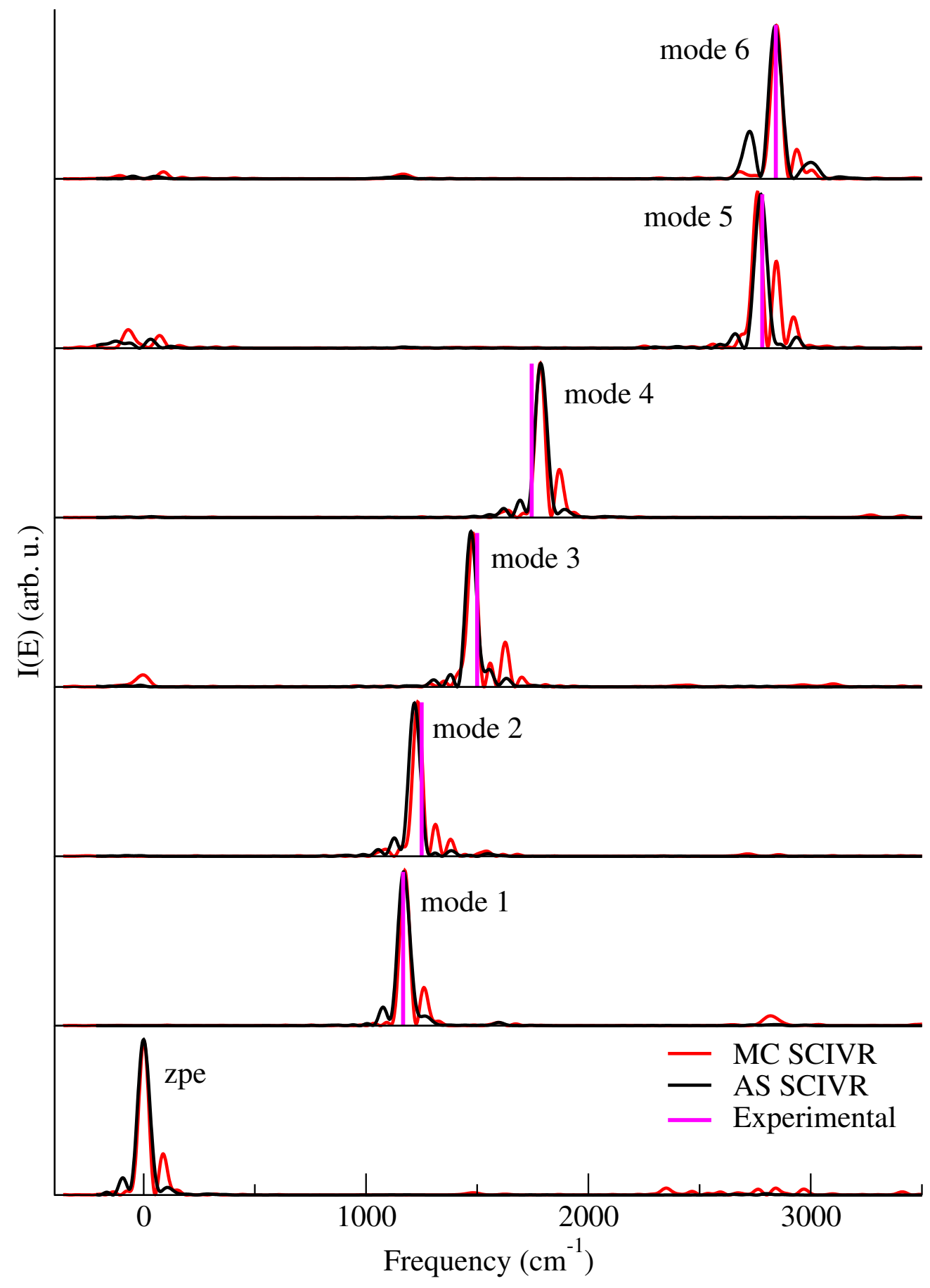

FIG. 4. MC-SCIVR (red) and AS-SCIVR (black) spectra of formaldehyde from 25000 au long trajectories. Experimental values (magenta) are taken from [78]. 
TABLE III. Zero-point energy and fundamental frequency for $\mathrm{H}_{2} \mathrm{CO}$. The full width at half maximum values are reported in parenthesis. Experimental values (Expt) are from [78]. MAE stands for mean absolute error. All values are in $\mathrm{cm}^{-1}$.

\begin{tabular}{c|c|c|c}
\hline Mode & Expt & MC SCIVR & AS SCIVR \\
\hline \hline zpe & - & $5862(51)$ & $5714(62)$ \\
1 & 1167 & $1174(51)$ & $1170(63)$ \\
2 & 1250 & $1232(49)$ & $1218(59)$ \\
3 & 1500 & $1482(50)$ & $1472(58)$ \\
4 & 1745 & $1784(50)$ & $1786(62)$ \\
5 & 2782 & $2760(50)$ & $2776(64)$ \\
6 & 2843 & $2846(57)$ & $2840(67)$ \\
\hline MAE & - & 18 & 19 \\
\hline
\end{tabular}

the MC-SCIVR signals are characterized by a small side peak, which is blue shifted by about 50 wavenumber with respect to the main one. Since $\mathrm{H}_{2} \mathrm{CO}$ does not have any low frequency (i.e. $\leq 100 \mathrm{~cm}^{-1}$ ) modes, the side peaks cannot represent the fingerprint of any combination of vibrational levels. They are instead a simulation artifact due to a slow and almost inappreciable rotation of the molecule during the trajectory, which is known to split the vibrational signal into two components. The effect is twofold: On the one hand the vibrational estimate provided by the MC-SCIVR method is less reliable; on the other hand, the two signals are narrower than the expected single one. As for the AS-SCIVR simulations of $\mathrm{H}_{2} \mathrm{CO}$, we point out that they provide better defined spectroscopic signals.

\section{Glycine (GLY)}

For small molecules, single-trajectory AS-SCIVR simulations provide just a slight improvement over the MC-SCIVR results, as it was indeed expected. However, the complexity of the spectrum increases as the system gets larger. Biological systems, for instance, range from the small glycine amino acid, made of 10 atoms, to functional aggregates made of thousands of atoms. For such systems any improvement in precision of the simulations may be crucial for a correct interpretation of the spectroscopic insights. For this reason, we ap- 
plied AS-SCIVR to glycine. This amino acid, made of 10 atoms, has already been studied by means of MC-SCIVR, therefore we are able to assess AS-SCIVR results against both experimental data and previous semiclassical state-of-the-art calculations.

Glycine is a floppy molecule with low interconversion barriers between conformers. Perturbing the actual Hamiltonian with an AS procedure may result in an undesired change of conformer during the AS run. To prevent this to happen we decided to adopt a longer switching trajectory $(35000 \mathrm{au})$ and to start the switching trajectory from the equilibrium momentum giving all modes harmonic momenta, i.e.

$$
p_{i}=\sqrt{\omega_{i}}
$$

where $\omega_{i}$ is the harmonic frequency of the $i$-th vibrational mass-scaled normal mode. This is a special case of the starting condition shown in Equation 9, in which $n_{i}=0, \zeta_{i}=-1 / 4$ $\forall i$.

Finally, previous experience with this amino acid suggested to assign zero energy to the first vibrational normal mode. This mode is a low-frequency internal rotation that sits along the conformer change coordinate playing a pivotal role in the conformational change of the molecule. However, for conservation of the action variables during the AS process, we could not set the momentum of the first vibrational mode to zero, in which case we would have lost the initial harmonic quantization. This was done only at the end of the AS run and preliminary to the SC dynamics. In this way, any spurious rotation was hindered.

With all these precautions in place, we were able to obtain the AS-SCIVR spectra at DFT/B3LYP aug-cc-pVDZ level of theory. The 35000 au-long switching trajectory from equilibrium conditions was followed by a 25000 au-long classical trajectory, whose starting conditions had no energy in the first vibrational normal mode. A selection of spectra thus obtained is compared with analogous MC-SCIVR spectra in Figure 5. Experimental [79, 80] and harmonic DFT/B3LYP/aug-cc-pVDZ frequencies are included. The spectral analysis is reported in Table IV. The AS-SCIVR spectra are sharper than the MC-SCIVR ones and much cleaner, as it can be appreciated from the zpe spectra in Figure 6. This is a consequence of the improved initial conditions given by $\mathrm{AS}$, with respect to the harmonicbased MC-SCIVR ones.

AS-SCIVR glycine signals generally feature a much less intense side peak at lower frequency. This is present in the zpe spectrum and in the plots of several fundamentals. 
Given the reduced intensity and its position, this is likely to be due to the coupling with a rotational state. This coupling is an effect of having evolved the switching trajectory in normal modes. Normal modes are rigorously defined only at the equilibrium geometry, so the back-and-forth between normal modes and Cartesian coordinates (although necessary) introduces some degree of vibro-rotational coupling. The side peak is expected to disappear when employing thousands of multiple trajectories, a task not affordable with an "on-thefly" simulation. Clearly, as expected, there are some discrepancies between MC-SCIVR and AS-SCIVR results, but these are minor. Overall, AS-SCIVR simulations are more precise than MC-SCIVR ones while their accuracy is comparable, as demonstrated by the similar mean absolute error values of about $35 \mathrm{~cm}^{-1}$ (see Table IV). Finally we notice that the zpe values are in excellent agreement (i.e. within just 5 wavenumbers) with diffusion Monte Carlo calculations recently performed on a high-level analytical surface for glycine.[15]

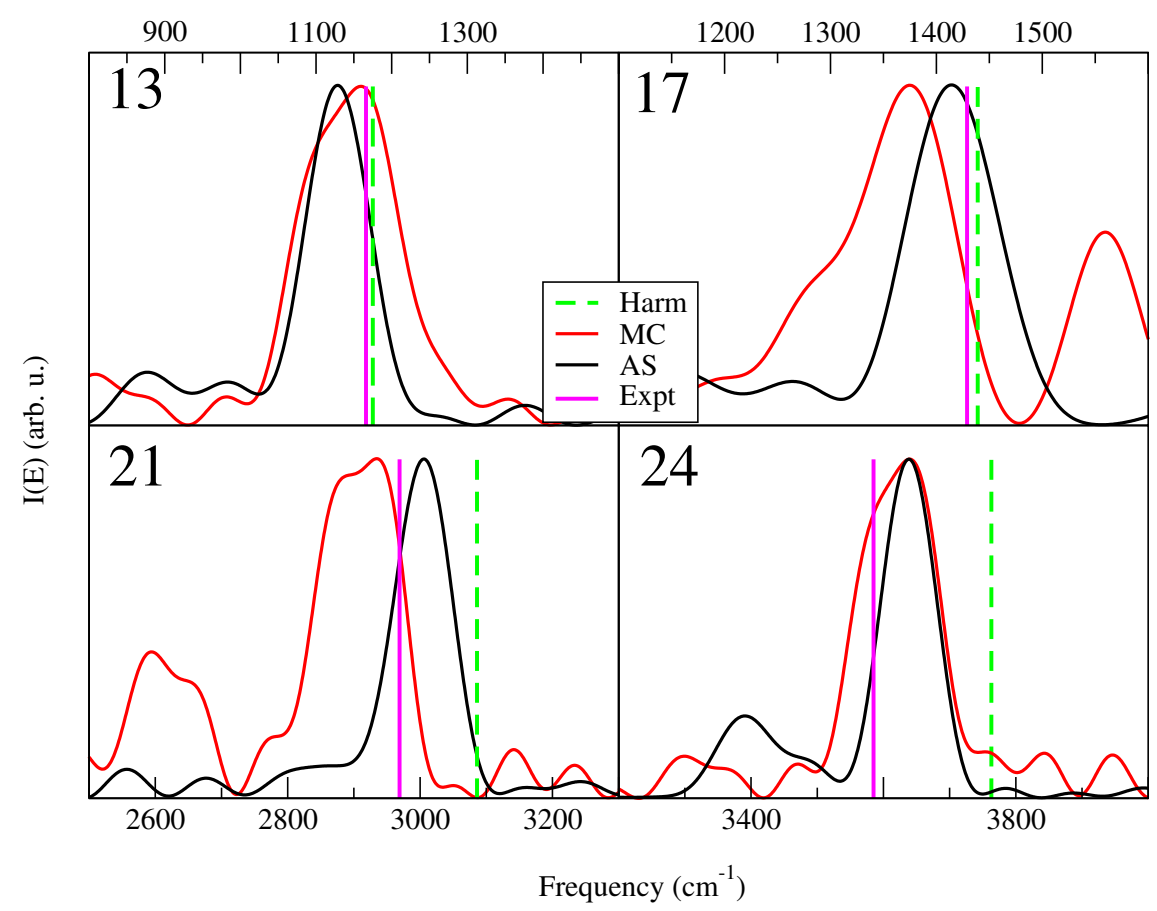

FIG. 5. Comparison between the Glycine peaks obtained through the different methods, at DFT/B3LYP aug-cc-pvDZ level of theory. In black the ones obtained with AS-SCIVR; in red the MC-SCIVR ones; in magenta the experimental frequencies $[79,80]$ and in green the harmonic frequencies 
TABLE IV. ZPE energies and transition frequencies with FWHM values in parenthesis for glycine spectra obtained with MC SCIVR and AS SCIVR, compared with harmonic (DFT/B3LYP augcc-pvDZ) and experimental values. [79, 80] The "Mean" row reports the average FWHM value.

\begin{tabular}{|c|c|c|c|c|}
\hline Mode & MC SCIVR / $\mathrm{cm}^{-1}$ & AS SCIVR / $\mathrm{cm}^{-1}$ & Harm $/ \mathrm{cm}^{-1}$ & Expt. $/ \mathrm{cm}^{-1}$ \\
\hline zpe & $17158(145)$ & $17152(96)$ & 17363 & - \\
\hline 1 & $99(107)$ & $72(92)$ & 59 & - \\
\hline 2 & $181(126)$ & $203(96)$ & 208 & 204 \\
\hline 3 & $229(131)$ & $261(93)$ & 249 & 250 \\
\hline 4 & $450(150)$ & $461(99)$ & 457 & 458 \\
\hline 5 & $499(146)$ & $495(93)$ & 510 & 500 \\
\hline 6 & $569(120)$ & $665(134)$ & 628 & 615 \\
\hline 7 & $667(137)$ & $596(93)$ & 647 & 619 \\
\hline 8 & $780(133)$ & $738(99)$ & 815 & 801 \\
\hline 9 & $776(69)$ & $732(95)$ & 906 & 907 \\
\hline 10 & $914(150)$ & $904(115$ & 911 & 883 \\
\hline 11 & $1103(156)$ & $1071(103)$ & 1120 & 1101 \\
\hline 12 & $1106(130)$ & $1115(100)$ & 1158 & 1136 \\
\hline 13 & $1159(153)$ & $1129(99)$ & 1175 & 1166 \\
\hline 14 & $1243(150)$ & $1266(98)$ & 1294 & 1297 \\
\hline 15 & $1299(147)$ & $1329(97)$ & 1371 & 1340 \\
\hline 16 & 1339 (151) & $1358(100)$ & 1384 & 1405 \\
\hline 17 & $1375(117)$ & $1414(102)$ & 1439 & 1429 \\
\hline 18 & $1628(86)$ & $1606(108)$ & 1656 & 1608 \\
\hline 19 & $1776(154)$ & $1778(92)$ & 1804 & 1779 \\
\hline 20 & 2945 (142) & $3005(98)$ & 3049 & 2943 \\
\hline 21 & 2934 (149) & $3006(101)$ & 3086 & 2969 \\
\hline 22 & $3316(161)$ & $3422(105)$ & 3495 & 3359 \\
\hline 23 & 3327 (92) & $3463(81)$ & 3569 & 3410 \\
\hline 24 & 3640 (142) & $3638(96)$ & 3736 & 3585 \\
\hline Mean & $-(133.76)$ & $-(99.40)$ & - & - \\
\hline MAE & 35.87 & 35.43 & 41.61 & - \\
\hline
\end{tabular}




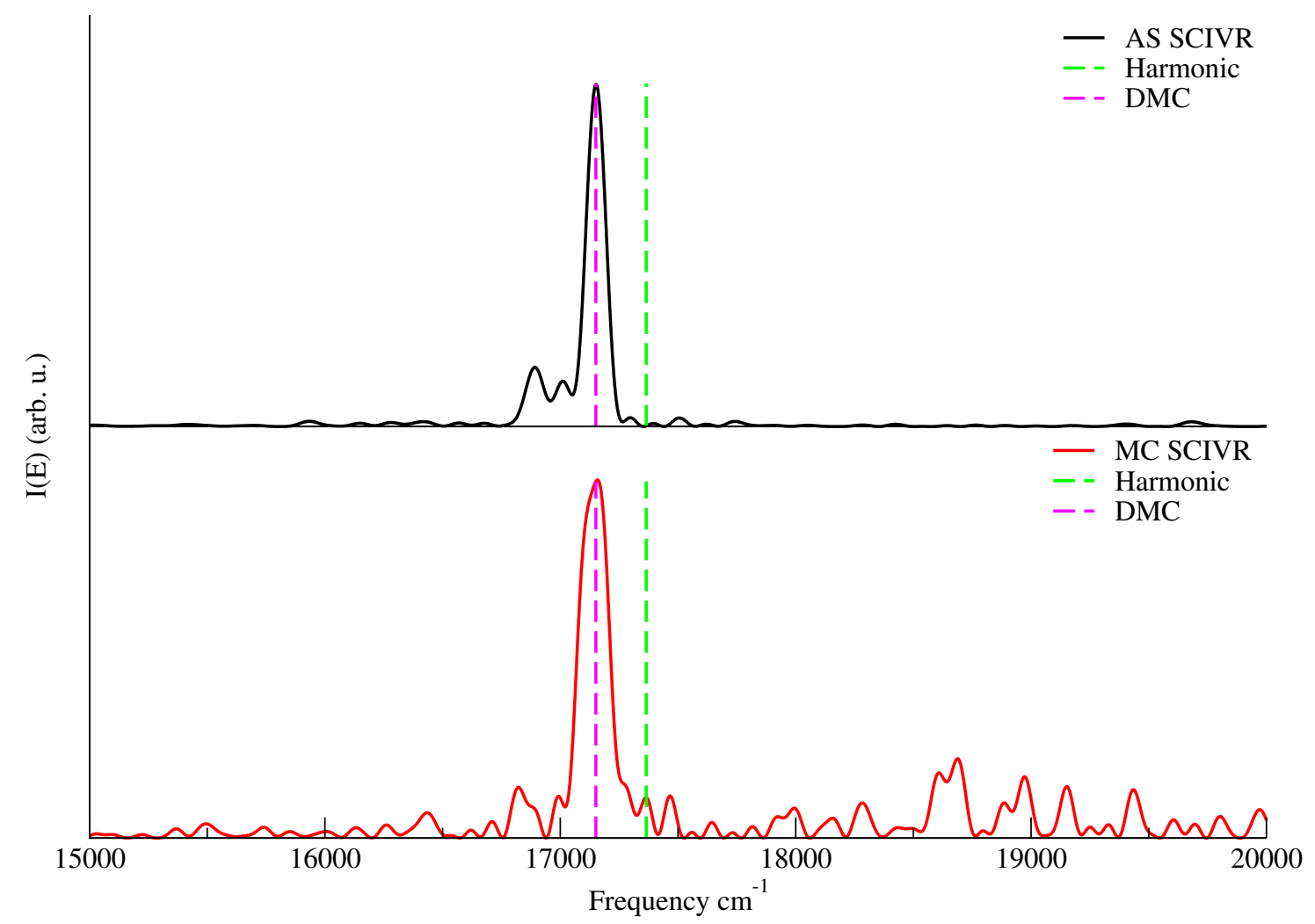

FIG. 6. Glycine zero point energy power spectrum peak. In black: AS-SCIVR zpe spectrum, obtained with a 25000 au classical trajectory at DFT/B3LYP aug-cc-pVDZ level of theory; in red: the analogous MC-SCIVR spectrum. The harmonic zpe is in dashed green, while the Diffusion Monte Carlo (DMC) one is in dashed magenta.[15]

\section{Proline}

After the encouraging results obtained with glycine, AS SCIVR was applied to the 17atom amino acid proline. Proline has a peculiar structure which makes it an immino acid rather than an amino acid. An experimental IR spectrum collected by Adamowicz et al. was not immediate to assign and triggered a lot of investigation and speculation about the conformers contributing to the spectrum. [60] First of all, four lower-energy conformers were isolated from a total of 15 minima at DFT/B3LYP/aug-cc-pVDZ level of theory. Of these, two presented a "type I" geometry (with NH---O internal hydrogen bond) and the other two a "type II" geometry (with N---HO internal hydrogen bond). These four geometries were considered the main candidates as contributors to the spectral features and were re-optimized at MP2/aug-cc-pVDZ level of theory. 
The main features of the experimental spectrum are the $3559 \mathrm{~cm}^{-1}$ peak - assigned to a type I OH stretch - and a double peak at 1789 and $1766 \mathrm{~cm}^{-1}$, which suggested to Adamowicz and co-authors that two type I conformers were present in the experiment. However, this was not confirmed by theoretical studies focused on the relative energy stability of the conformers. These show that a type II conformer is the most stable, at all level of theory investigated (DFT, MP2, MP4 and $\operatorname{CCSD}(\mathrm{T}))$. Therefore, in the same paper, the authors concluded that another possibility was that the experimental spectrum was actually due to the presence of one type II and one type I conformer, close enough in energy to be both populated at the experimental conditions $(14 \mathrm{~K})$.

The issue of the assignment of experimental IR spectrum of proline has not been definitively settled down yet. Since semiclassical spectroscopy has already been proven to be a reliable tool for spectral assignment, as a final demonstration of "on-the-fly" AS SCIVR, we present here the main features of the vibrational power spectrum of proline most stable conformer.

Following Adamowicz's investigation, we optimized the type II conformer at DFTD/B3LYP/aug-cc-pVDZ level of theory. The resulting geometry, reported in Figure 7, was employed as an equilibrium geometry for the subsequent work. The DFT-D/B3LYP functional allowed us to keep the computational effort affordable while accounting for the long range interaction with the semiempirical diffusion terms.

Both the switching and classical trajectories were run for 25000 au at that same level of theory and basis set. Similarly to the case of glycine, the switching trajectory was initiated at equilibrium atomic positions with harmonic momenta (i.e. $\xi=-1 / 4$ ). To further reduce the computational effort, the number of Hessian matrices to be computed was reduced from 2500 to 149, according to the Hessian Database procedure adopted using a threshold of $2 \times 10^{-1}$

The 10 highest frequencies as obtained with AS SCIVR are listed in Table V. A full list of frequencies can be found in the Supplemental Material. The semiclassical spectra of mode 36 ( $\mathrm{C}=\mathrm{O}$ stretch), 44 ( $\mathrm{OH}$ stretch), and 45 ( $\mathrm{NH}$ stretch) are instead shown in Figure 8, compared with the quasi-classical trajectory (QCT) spectra for the same mode and the harmonic frequencies. 


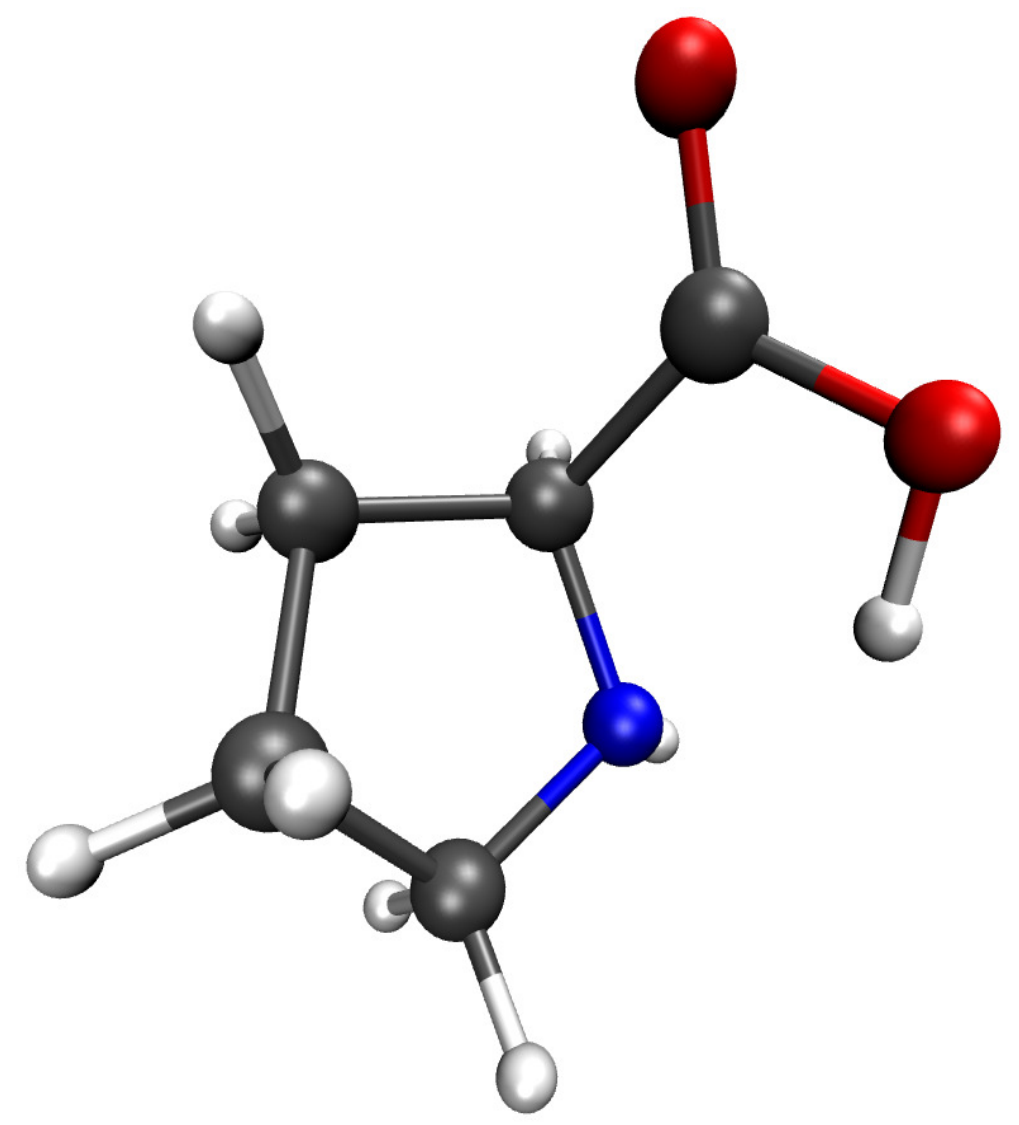

FIG. 7. Trans Proline

The QCT spectrum of the $j$-th vibrational normal mode has been evaluated as[81, 82]

$$
I_{j}(E)=\frac{1}{2 T}\left|\int_{0}^{T} d t e^{i E t / \hbar} p_{j}(t)\right|^{2},
$$

where $T$ is the total classical trajectory time, $E$ is the energy, and $p_{j}(t)$ is the linear momentum of the $j$-th vibrational normal mode at time $t . I_{j}(E)$ is a classical spectrum that cannot describe any quantum effects like the zero-point energy, anharmonic overtones, quantum resonances and interferences. This drawback is partially compensated by the reduced complexity of the QCT spectrum, which makes it a suitable candidate for a computationallycheap assessment of the AS-SCIVR spectra quality.

The comparison in Figure 8 shows that AS-SCIVR specta are sharper than the QCT 
TABLE V. High-range harmonic and semiclassical frequencies (FWHM values in parenthesis) in $\mathrm{cm}^{-1}$ for proline (DFT-D/B3LYP/aug-cc-pVDZ level of theory).

\begin{tabular}{c|c|lc}
\hline Mode & Vibration & Harm AS-SCIVR (FWHM) \\
\hline \hline 45 & NH str & 3560 & $3461(50.51)$ \\
44 & OH str & 3355 & $3329(51.90)$ \\
43 & CH str & 3135 & $3071(52.24)$ \\
42 & CH str & 3117 & $3060(47.46)$ \\
41 & CH str & 3086 & $2970(62.28)$ \\
40 & CH str & 3074 & $2949(53.86)$ \\
39 & CH str & 3057 & $2938(55.00)$ \\
38 & CH str & 3050 & $3017(45.78)$ \\
37 & CH str & 3003 & $2911(67.00)$ \\
36 & C=O str & 1824 & $1799(47.20)$ \\
\hline ZPE & - & 31843 & $31362(47.98)$ \\
\hline Avg & - & - & $-(47.98)$ \\
\hline
\end{tabular}

ones, but also "noisier" presenting several side peaks. These can be due to ro-vibrational coupling, as already illustated in the case of glycine, or actual "noise" as an effect of the large dimensionality (45 degrees of freedom) of the system. As the system grows in dimensionality, the signal-to-noise ratio is reduced due to the reduction of the full-dimensional overlap integral of Equation 1. The full-dimensional simulation of 17-atom proline is clearly affected by this issue, but the AS-SCIVR technique still manages to collect a clear signal.

Finally, we notice that a remarkable red shift of the AS-SCIVR signal associated to the $\mathrm{OH}$ stretch with respect to its classical (QCT) counterpart, based on the same trajectory, is present. This is a clue that an accurate spectroscopic description of the $\mathrm{OH}$ stretch and associated hydrogen bond necessitate a quantum treatment.

\section{SUMMARY AND PERSPECTIVES}

In this work we have extended the range of application of the AS-SCIVR technique by implementing it for "on-the-fly" simulations. We have successfully tested it on a set 


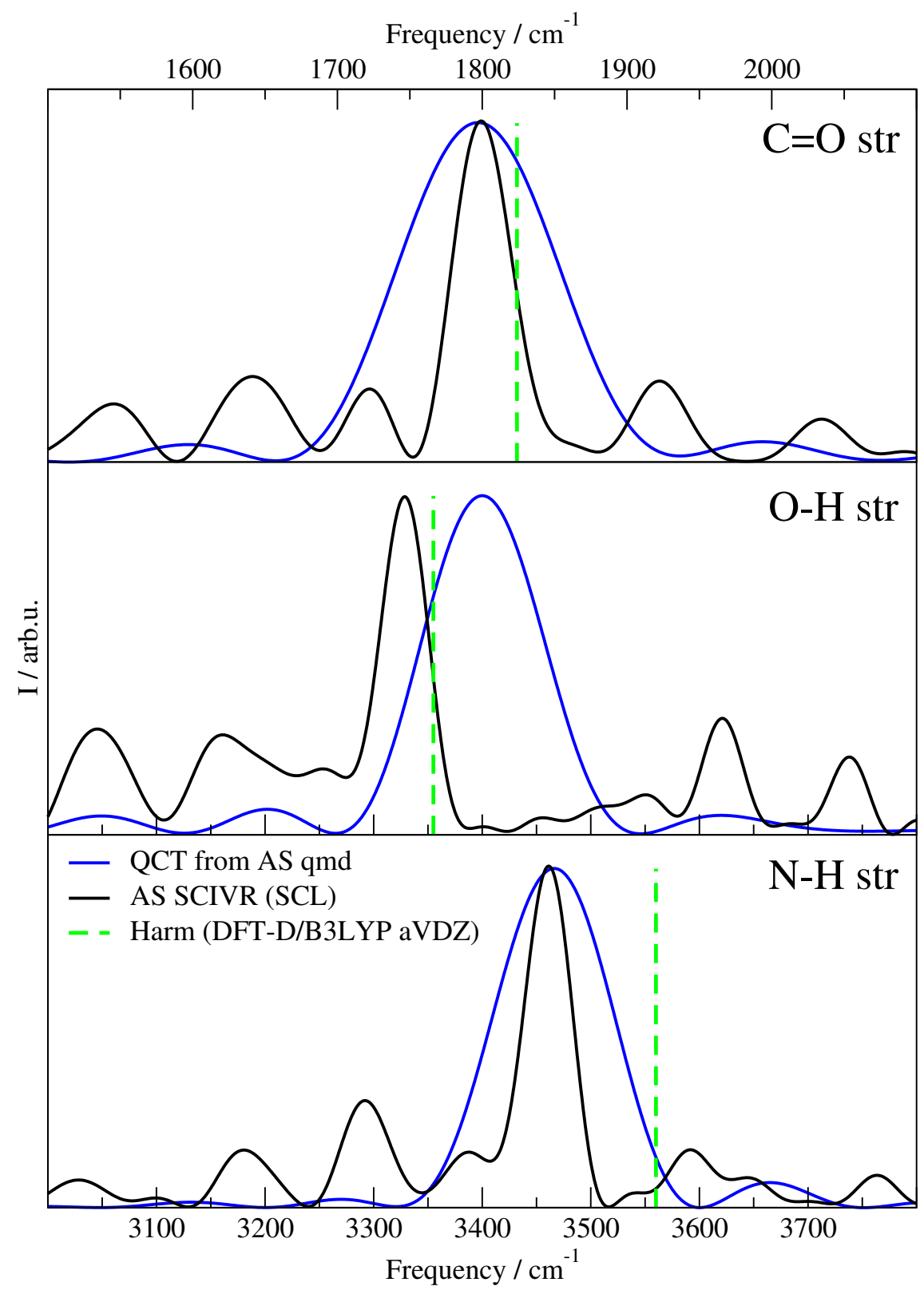

FIG. 8. Comparison between the Proline peaks obtained through the different methods, at DFTD/B3LYP aug-cc-pvDZ level of theory. In black the ones obtained with AS SCIVR; in blue the quasi-classical ones and in dashed green the harmonic frequencies

of molecules up to glycine for which semiclassical results obtained with other techniques were already available. Finally, we have presented a new application to the global minimum conformer of the proline amino acid showing the general importance of employing a quantum approach in spectroscopy simulations. This is a crucial aspect especially for molecular systems, like proline, whose vibrational spectroscopy is still debated. 
"On-the-fly" AS SCIVR has permitted to confirm the main advantages of the method already demonstrated for small molecules with analytical PES available.[48] Specifically, the adiabatic switching pre-treatment of the trajectory leads generally to more precise, i.e. with narrower peaks, and accurate results. AS SCIVR allows one to push the limit for a full-dimensional calculation, and we were able to treat 17-atom proline in full dimensionality.

There are two potential drawbacks of the technique which anyway did not hamper the simulations here presented. One is due to the necessity to generate a single trajectory when solving the electronic problem "on-the-fly". It may happen that at the end of the AS procedure the energy content of the trajectory is below the zero-point energy of the system, in which case the final frequency estimate could be slightly shifted towards the harmonic estimate. We found this to have a very minor impact in our simulations. The second issue is related to the possibility that a small amount of ro-vibrational coupling during the preliminary AS run is accidentally introduced. This has produced some minor side peaks that were not a threat to the correct assignment of spectral features. The problem could be partially eased by implementing the adiabatic switching procedure in internal coordinates, [46] which is a possible future development.

We conclude with a few remarks about the open spectroscopic problem regarding proline. We briefly discussed about the signal-to-noise issue, which we know it can be overcome by interfacing AS SCIVR with the DC-SCIVR technique. This is something we plan to do in a follow-up work dedicated to proline to try to clarify the spectroscopic assignment of this elusive amino acid. Experiments are not conclusive on this aspect, so we plan to calculate the spectra of several low-energy conformers which can contribute to the IR spectrum of proline. Given the high dimensionality and complexity of the problem, semiclassical techniques and AS SCIVR specifically appear to be suitable to solve this difficult problem. Finally, the work here presented and its envisaged developments demonstrate that new frontiers in the theoretical spectroscopy of biological species can be achieved, providing the necessary quantum description to correctly characterize the vibrational motion and related properties of biomolecular systems. 


\section{SUPPLEMENTARY MATERIAL}

The supplementary material file contains the starting geometries and velocities for all simulations, and complete MC-SCIVR and AS-SCIVR spectra of $\mathrm{H}_{2} \mathrm{CO}$, glycine, and proline.

\section{ACKNOWLEDGMENTS}

The authors acknowledge financial support from the European Research Council [Grant Agreement No. (647107) — SEMICOMPLEX —ERC-2014-CoG] under the European Union's Horizon 2020 research and innovation program, and from the Italian Ministry of Education, University, and Research (MIUR) (FARE Program No. R16KN7XBRB-project QURE). G.B. thanks INPS for funding his Ph.D. scholarship.

\section{DATA AVAILABILITY}

The data that support the findings of this study are available from the corresponding author upon reasonable request. 
[1] W. D. Cornell, P. Cieplak, C. I. Bayly, I. R. Gould, K. M. Merz, D. M. Ferguson, D. C. Spellmeyer, T. Fox, J. W. Caldwell, and P. A. Kollman, J. Am. Chem. Soc. 118, 2309 (1996).

[2] J. Wang, P. Cieplak, and P. A. Kollman, J. Comput. Chem. 21, 1049 (2000).

[3] J. A. Maier, C. Martinez, K. Kasavajhala, L. Wickstrom, K. E. Hauser, and C. Simmerling, J. Chem. Theory Comput. 11, 3696 (2015).

[4] R. B. Best, X. Zhu, J. Shim, P. E. Lopes, J. Mittal, M. Feig, and A. D. MacKerell Jr, J. Chem. Theory Comput. 8, 3257 (2012).

[5] P. Ren and J. W. Ponder, J. Comput. Chem. 23, 1497 (2002).

[6] J. W. Ponder, C. Wu, P. Ren, V. S. Pande, J. D. Chodera, M. J. Schnieders, I. Haque, D. L. Mobley, D. S. Lambrecht, R. A. DiStasio Jr, et al., J. Phys. Chem. B 114, 2549 (2010).

[7] Y. Shi, Z. Xia, J. Zhang, R. Best, C. Wu, J. W. Ponder, and P. Ren, J. Chem. Theory Comput. 9, 4046 (2013).

[8] A. Esser, S. Belsare, D. Marx, and T. Head-Gordon, Phys. Chem. Chem. Phys. 19, 5579 (2017).

[9] C. Zhang, C. Lu, Z. Jing, C. Wu, J.-P. Piquemal, J. W. Ponder, and P. Ren, J. Chem. Theory Comput. 14, 2084 (2018).

[10] R. Ramakrishnan, P. O. Dral, M. Rupp, and O. A. von Lilienfeld, J. Chem. Theory Comput. 11, 2087 (2015).

[11] S. Chmiela, H. E. Sauceda, K.-R. Müller, and A. Tkatchenko, Nat. Commun. 9, 3887 (2018).

[12] H. E. Sauceda, S. Chmiela, I. Poltavsky, K.-R. Müller, and A. Tkatchenko, J. Chem. Phys. 150, 114102 (2019).

[13] A. P. Bartok, S. De, C. Poelking, N. Bernstein, J. R. Kermode, G. Csanyi, and M. Ceriotti, Sci. Adv. 3 (2017).

[14] R. Conte, C. Qu, P. L. Houston, and J. M. Bowman, J. Chem. Theory Comput. 16, 3264 (2020).

[15] R. Conte, P. L. Houston, C. Qu, J. Li, and J. M. Bowman, J. Chem. Phys. 153, 244301 (2020).

[16] A. Nandi, C. Qu, P. L. Houston, R. Conte, and J. M. Bowman, J. Chem. Phys. 154, 051102 (2021). 
[17] C. Qu, P. L. Houston, R. Conte, A. Nandi, and J. M. Bowman, J. Phys. Chem. Lett. 12, $4902(2021)$.

[18] R. Conte, G. Botti, and M. Ceotto, Vib. Spectrosc. 106, 103015 (2020).

[19] D. R. Moberg and A. W. Jasper, J. Chem. Theory Comput. 17, 5440 (2021).

[20] W. H. Miller, Proc. Natl. Acad. Sci. USA 102, 6660 (2005).

[21] W. H. Miller, J. Phys. Chem. A 105, 2942 (2001).

[22] E. J. Heller, Acc. Chem. Res. 14, 368 (1981).

[23] K. G. Kay, Chem. Phys. 322, 3 (2006).

[24] E. Pollak, "The Semiclassical Initial Value Series Representation of the Quantum Propagator," in Quantum Dynamics of Complex Molecular Systems (Springer Berlin Heidelberg, Berlin, Heidelberg, 2007) pp. 259-271.

[25] D. V. Shalashilin and M. S. Child, J. Chem. Phys. 115, 5367 (2001).

[26] F. Grossmann, J. Chem. Phys. 125 (2006).

[27] R. Conte and M. Ceotto, Quantum Chemistry and Dynamics of Excited States: Methods and Applications , 595 (2020).

[28] X. Ma, G. Di Liberto, R. Conte, W. L. Hase, and M. Ceotto, J. Chem. Phys. 149, 164113 (2018).

[29] T. Begušić and J. Vaníček, J. Chem. Phys. 153, 184110 (2020).

[30] M. Ceotto, S. Atahan, G. F. Tantardini, and A. Aspuru-Guzik, J. Chem. Phys. 130, 234113 (2009).

[31] A. L. Kaledin and W. H. Miller, J. Chem. Phys. 118, 7174 (2003).

[32] A. L. Kaledin and W. H. Miller, J. Chem. Phys. 119, 3078 (2003).

[33] N. De Leon and E. J. Heller, J. Chem. Phys. 78, 4005 (1983).

[34] M. Ceotto, G. Di Liberto, and R. Conte, Phys. Rev. Lett. 119, 010401 (2017).

[35] M. Gandolfi, A. Rognoni, C. Aieta, R. Conte, and M. Ceotto, J. Chem. Phys. 153, 204104 (2020).

[36] M. Cazzaniga, M. Micciarelli, F. Moriggi, A. Mahmoud, F. Gabas, and M. Ceotto, J. Chem. Phys. 152, 104104 (2020).

[37] G. Bertaina, G. Di Liberto, and M. Ceotto, J. Chem. Phys. 151, 114307 (2019).

[38] G. Di Liberto, R. Conte, and M. Ceotto, J. Chem. Phys. 148, 014307 (2018).

[39] G. Di Liberto, R. Conte, and M. Ceotto, J. Chem. Phys. 148, 104302 (2018). 
[40] M. Buchholz, F. Grossmann, and M. Ceotto, J. Chem. Phys. 147, 164110 (2017).

[41] M. Buchholz, E. Fallacara, F. Gottwald, M. Ceotto, F. Grossmann, and S. D. Ivanov, Chem. Phys. 515, 231 (2018).

[42] M. Buchholz, F. Grossmann, and M. Ceotto, J. Chem. Phys. 148, 114107 (2018).

[43] Q. Sun, J. M. Bowman, and B. Gazdy, J. Chem. Phys. 89, 3124 (1988).

[44] J. Huang, J. J. Valentini, and J. T. Muckerman, J. Chem. Phys. 102, 5695 (1995).

[45] C. Qu and J. M. Bowman, J. Phys. Chem. A 120, 4988 (2016).

[46] T. Nagy and G. Lendvay, J. Phys. Chem. Lett. 8, 4621 (2017).

[47] A. Bose and N. Makri, J. Chem. Theory Comput. 14, 5446 (2018).

[48] R. Conte, L. Parma, C. Aieta, A. Rognoni, and M. Ceotto, J. Chem. Phys. 151, 214107 (2019).

[49] F. Gabas, G. Di Liberto, R. Conte, and M. Ceotto, Chem. Sci. 9, 7894 (2018).

[50] C. Aieta, M. Micciarelli, G. Bertaina, and M. Ceotto, Nat. Comm. 11, 4384 (2020).

[51] C. Aieta, G. Bertaina, M. Micciarelli, and M. Ceotto, J. Chem. Phys. 153, 214117 (2020).

[52] F. Gabas, R. Conte, and M. Ceotto, J. Chem. Theory Comput. 13, 2378 (2017).

[53] N. L. Summers and M. Karplus, J. Mol. Biol. 216, 991 (1990).

[54] M. M. MacArthur and J. M. Thornton, J. Mol. Biol. 218, 397 (1991).

[55] J. Richardson and D. Richardson, Science 240, 1648 (1988).

[56] E. Czinki and A. G. Császár, Chem. Eur. J. 9, 1008 (2003).

[57] J. Schmidt and S. R. Kass, J. Phys. Chem. A 117, 4863 (2013).

[58] J. W. Silzel, T. A. Murphree, R. K. Paranji, M. M. Guttman, and R. R. Julian, J. Am. Soc. Mass Spectrom., 1974 (2020).

[59] I. Reva, S. Stepanian, A. Plokhotnichenko, E. Radchenko, G. Sheina, and Y. Blagoi, J. Mol. Struct. 318, 1 (1994).

[60] S. G. Stepanian, I. D. Reva, E. D. Radchenko, and L. Adamowicz, J. Phys. Chem. A 105, 10664 (2001).

[61] F. Gabas, G. Di Liberto, and M. Ceotto, J. Chem. Phys. 150, 224107 (2019).

[62] M. Micciarelli, F. Gabas, R. Conte, and M. Ceotto, J. Chem. Phys. 150, 184113 (2019).

[63] F. Gabas, R. Conte, and M. Ceotto, J. Chem. Theory Comput. 16, 3476 (2020).

[64] M. Micciarelli, R. Conte, J. Suarez, and M. Ceotto, J. Chem. Phys. 149, 064115 (2018).

[65] W. H. Miller, J. Chem. Phys. 53, 1949 (1970). 
[66] Y. Elran and K. Kay, J. Chem. Phys. 110, 3653 (1999).

[67] Y. Elran and K. Kay, J. Chem. Phys. 110, 8912 (1999).

[68] E. J. Heller, J. Chem. Phys. 75, 2923 (1981).

[69] M. F. Herman and E. Kluk, Chem. Phys. 91, 27 (1984).

[70] K. G. Kay, J. Chem. Phys. 100, 4377 (1994).

[71] K. G. Kay, J. Chem. Phys. 101, 2250 (1994).

[72] K. G. Kay, J. Chem. Phys. 100, 4432 (1994).

[73] R. Conte, F. Gabas, G. Botti, Y. Zhuang, and M. Ceotto, J. Chem. Phys. 150, 244118 (2019).

[74] M. Ceotto, S. Atahan, S. Shim, G. F. Tantardini, and A. Aspuru-Guzik, Phys. Chem. Chem. Phys. 11, 3861 (2009).

[75] R. Conte, A. Aspuru-Guzik, and M. Ceotto, J. Phys. Chem. Lett. 4, 3407 (2013).

[76] L. D. Landau and E. M. Lifshitz, Mechanics (Elsevier, 1982).

[77] M. Valiev, E. Bylaska, N. Govind, K. Kowalski, T. Straatsma, H. Van Dam, D. Wang, J. Nieplocha, E. Apra, T. Windus, and W. de Jong, Comput. Phys. Commun. 181, 1477 (2010).

[78] T. Nakanaga, S. Kondo, and S. Saeki, J. Chem. Phys. 76, 3860 (1982).

[79] S. G. Stepanian, I. D. Reva, E. D. Radchenko, M. T. S. Rosado, M. L. T. S. Duarte, R. Fausto, and L. Adamowicz, J. Phys. Chem. A 102, 1041 (1998).

[80] G. Bazsó, G. Magyarfalvi, and G. Tarczay, J. Phys. Chem. A 116, 10539 (2012).

[81] A. Rognoni, R. Conte, and M. Ceotto, J. Chem. Phys. 154, 094106 (2021).

[82] A. Rognoni, R. Conte, and M. Ceotto, Chem. Sci. 12, 2060 (2021). 